\title{
Statistical Analysis of Voltage Unbalance Emission Due to Asymmetrical Loads in Three-Phase Power Systems
}

\author{
Diego Bellan \\ Department of Electronics, Information and Bioengineering \\ Politecnico di Milano, \\ Piazza Leonardo da Vinci 32, Milan, 20133 \\ Italy \\ Received: July 17, 2021. Revised: January 11, 2022. Accepted: January 29, 2022. \\ Published: February 25, 2022.
}

\begin{abstract}
This paper investigates the statistical properties of the voltage unbalance factor in a three-phase system due to an asymmetrical three-phase load with uncertain parameters. The parameters of the three-phase load are treated as random variables with Gaussian distribution. Random asymmetry in the three-phase load results in random values of the voltage unbalance factor. The probability density function, the cumulative distribution function, the mean value and the variance of the voltage unbalance factor are derived in closed form and numerically validated. The obtained results are useful to provide a quantitative description of possible effects of asymmetry in a three-phase load such as the connection of a large single-phase load.
\end{abstract}

Keywords-Asymmetrical three-phase loads, statistical analysis, voltage unbalance factor.

\section{INTRODUCTION}

$\mathrm{T}$ HREE-phase power systems under steady-state sinusoidal conditions are usually analyzed by resorting to the wellknown symmetrical component transformation (SCT) [1]-[3]. This approach is a special case of the more general approach consisting in the modal analysis of circuits. Roughly speaking, modal analysis of circuits consists in a proper matrix transformation of voltage/current vectors leading to diagonal impedance/admittance matrices. Thus, the transformed system can be seen as a collection of uncoupled circuits whose solution can be readily evaluated. The SCT yields three uncoupled circuits (i.e., the positive, negative, and zero sequence circuits) under the fundamental assumption of a three-phase system with perfect structural symmetry between the three phases, i.e., the circuit parameters of the three phases (e.g., resistance, capacitance, self and mutual inductance) must be equal. In practical three-phase power systems, however, perfect symmetry of the three phases cannot be guaranteed.
Indeed, the two main reasons leading to three-phase asymmetry are related to the lines and to the loads [4]-[9]. In fact, three-phase lines are usually transposed such that the three conductors can be regarded as geometrically equivalent along the whole length of the line. As far as the loads are concerned, however, the use of large single-phase loads (e.g., high-speed railway lines) can result in a significant asymmetry in a three-phase load. The lack of symmetry in a three-phase load results in a mutual coupling between the sequence circuits after the symmetrical component transformation.

In an ideal three-phase power system the voltage source consists only in the positive sequence component. Therefore, in a symmetrical system, the negative and zero sequence circuits are not excited. If the symmetry assumption is not met, however, coupling between sequence circuits results in injection of current also in the negative and zero sequence circuit. In this process the positive sequence circuit acts as the source of emission, and the negative and zero sequence circuits represent the victims. In many cases, power systems consist only in the interconnection of components with three wires. In such case, the zero-sequence circuit is an open circuit. Thus, the victim of the emission is the negative sequence circuit only. Emission of the positive sequence circuit on the negative sequence circuit due to lack of system symmetry is called voltage unbalance emission [10]-[15].

In practical applications it is of paramount importance to limit such phenomenon because it has adverse effects on both supply utilities and customer installations. Three-phase rotating machines are the major victim under unbalanced supply voltages. In particular, the presence of voltage unbalance adversely affects the operation of three-phase induction motors in terms of high unbalanced phase currents, reduced motor torque and speed, increased noise and vibration. Additional heat in windings leads to reduction in the motor efficiency, thus demanding motor de-rating [14]-[15].

It is important to notice that voltage unbalance emission is one of the interference phenomena regarded as low-frequency conducted emission in electromagnetic compatibility (EMC) 
[16]-[17]. Indeed, EMC investigates electromagnetic phenomena starting from zero hertz. From this viewpoint, voltage unbalance emission is one of the main conducted lowfrequency phenomena.

Since in many cases of practical interest the asymmetry level in a three-phase load can be regarded as a small perturbation with respect to the ideal symmetrical condition, in this paper a weak-coupling approach between sequence circuits is proposed [18]. Analytical investigation of the problem provides the conditions to be met such that weakcoupling can be assumed. Thus, in case of weak coupling the emission from the positive to the negative sequence circuit can be readily evaluated through a simple and approximate equivalent circuit consisting in a current-controlled voltage source depending on the load asymmetry. Then, the approximate equivalent circuit is used to derive the statistical properties of the voltage unbalance factor due to an asymmetrical three-phase load with uncertain parameters. Indeed, by treating the load parameters as Gaussian random variables the statistics of the voltage unbalance factor are derived in closed form and numerically validated.

The paper is organized as follows. In Section II the symmetrical component transformation is recalled. In Section III, the circuit representation of load asymmetry under the assumption of weak coupling between positive and negative sequence circuits is derived, and an approximate expression for the voltage unbalance factor is provided. In Section IV the statistical analysis of the voltage unbalance factor is performed by deriving its probability density function, cumulative distribution function, mean value and variance. The obtained analytical results are numerically validated in Section V. Finally, concluding remarks are reported in Section VI.

\section{BACKGROUND: THE SYMMETRICAL COMPONENT TRANSFORMATION}

The conventional approach for the steady-state analysis of symmetrical three-phase power systems foresees the use of the Symmetrical Component Transformation (SCT) in the phasor domain. The main advantage of this approach consists in obtaining decoupled equations even if the three phases are coupled in the original variables. This approach is a special case of the more general modal analysis of coupled circuits. The three uncoupled modal circuits, called sequence circuits (i.e., positive, negative, and zero sequence circuits), can be readily solved in the sequence domain and the original variables can be recovered by inverse transformation.

The transformation matrix, in its rational form, is defined as

$$
\mathbf{S}=\frac{1}{\sqrt{3}}\left[\begin{array}{ccc}
1 & \alpha & \alpha^{2} \\
1 & \alpha^{2} & \alpha \\
1 & 1 & 1
\end{array}\right]
$$

where

$$
\alpha=e^{j \frac{2}{3} \pi}=-\frac{1}{2}+j \frac{\sqrt{3}}{2}
$$

and $\alpha^{2}=\alpha^{*}$, where asterisk denotes complex conjugate. The transformation matrix (1) is a Hermitian matrix, i.e., $\mathbf{S}^{-1}=$ $\mathbf{S}^{T *}$.

The SCT when applied to phasor voltages provides

$$
\left[\begin{array}{c}
V_{p} \\
V_{n} \\
V_{\mathbf{0}}
\end{array}\right]=\mathbf{S} \cdot\left[\begin{array}{c}
V_{a} \\
V_{b} \\
V_{c}
\end{array}\right]
$$

where $V_{p}, V_{n}$, and $V_{0}$ are the positive, negative, and zero sequence voltages. Of course, the same transformation applies to phasor currents.

Symmetrical three-phase passive components (i.e., lines and loads) can be described in terms of an impedance matrix with the following structure

$$
\mathbf{Z}=\left[\begin{array}{ccc}
Z & Z_{m} & Z_{m} \\
Z_{m} & Z & Z_{m} \\
Z_{m} & Z_{m} & Z
\end{array}\right]
$$

By defining the column vectors

$$
\mathbf{V}_{s}=\left[\begin{array}{c}
V_{p} \\
V_{n} \\
V_{0}
\end{array}\right], \quad \mathbf{I}_{s}=\left[\begin{array}{c}
I_{p} \\
I_{n} \\
I_{0}
\end{array}\right], \quad \mathbf{V}=\left[\begin{array}{c}
V_{a} \\
V_{b} \\
V_{c}
\end{array}\right], \quad \mathbf{I}=\left[\begin{array}{c}
I_{a} \\
I_{b} \\
I_{c}
\end{array}\right]
$$

the transformed current/voltage relationship for a symmetrical passive component can be written

$$
\mathbf{V}_{s}=\mathbf{S} \mathbf{Z S}-1 \mathbf{I}_{s}=\mathbf{Z}_{s} \mathbf{I}_{s}
$$

where

$$
\mathbf{Z}_{s}=\left[\begin{array}{ccc}
Z_{p} & 0 & 0 \\
0 & Z_{n} & 0 \\
0 & 0 & Z_{0}
\end{array}\right]
$$

and

$$
\begin{gathered}
Z_{p}=Z_{n}=Z-Z_{m} \\
Z_{0}=Z+2 Z_{m}
\end{gathered}
$$

The diagonal form of the sequence impedance matrix (7) leads to the above-mentioned uncoupled sequence circuits when the transformation is applied to the whole three-phase system.

\section{ASYMMETRICAL LOADS: WEAK COUPLING ANALYSIS}

In many cases of practical interest, the equivalent load of a three-phase power system can be modelled as three uncoupled impedances $Z_{a}, Z_{b}$, and $Z_{c}$. The power system is designed such that the three impedances are equal, but in practice a deviation from the nominal impedance level cannot be avoided.

By denoting as $Z$ the nominal value of the load impedances, 
the three load impedances can be written in terms of the arbitrary deviation (i.e., no symmetrical deviations are required) with respect to such nominal value:

$$
Z_{a}=Z+\delta Z_{a}, \quad Z_{b}=Z+\delta Z_{b}, \quad Z_{c}=Z+\delta Z_{c}
$$

By using the SCT defined in Section II, and by considering only the positive and negative sequence circuits (i.e., the common condition of a power system with three wires is considered), the voltage/current relationship (6) can be written as:

$$
\left[\begin{array}{l}
V_{p} \\
V_{n}
\end{array}\right]=\mathbf{Z}_{\text {sload }}\left[\begin{array}{l}
I_{p} \\
I_{n}
\end{array}\right]=\left[\begin{array}{cc}
Z & \delta Z_{n} \\
\delta Z_{p} & Z
\end{array}\right]\left[\begin{array}{l}
I_{p} \\
I_{n}
\end{array}\right]
$$

where

$$
\begin{aligned}
& \delta Z_{p}=\left(\delta Z_{a}+\alpha \delta Z_{b}+\alpha^{2} \delta Z_{c}\right) / 3 \\
& \delta Z_{n}=\left(\delta Z_{a}+\alpha^{2} \delta Z_{b}+\alpha \delta Z_{c}\right) / 3
\end{aligned}
$$

Notice that $\delta Z_{p}$ and $\delta Z_{n}$ are defined by expressions similar to $V_{p}$ and $V_{n}$ in (3), respectively (i.e., with $1 / 3$ instead of $1 / \sqrt{3}$ as a multiplicative factor).

Relationships (12)-(13) provide the coupling coefficients between the positive and negative sequence circuits due to an asymmetrical three-phase load. The remaining parts of the sequence circuits consist in the same line impedance $Z_{\text {line }}$ (since from (8) the positive and negative-sequence line impedances are equal), and the positive/negative components $E_{p}$ and $E_{n}$ of the three-phase source (see Fig. 1).

Voltage unbalance emission of the load consists in the voltage $V_{n}$ in Fig. 1 due to the coupling of the negative sequence circuit with the positive sequence circuit. A rigorous solution of the circuit in Fig. 1 can be obtained by solving a coupled problem. An approximate and simpler solution based on a weak coupling assumption, however, would be interesting for engineering applications. Indeed, the approximate procedure outlined in this paper can be readily extended to the case of a power system with more complicated structure and including several asymmetrical loads.

The problem in Fig. 1 can be seen as an interference problem where the source circuit consists in the positive sequence circuit, and the victim circuit consists in the negative sequence circuit. Assuming weak coupling means that the positive sequence current is not affected by the negative sequence circuit:

$$
I_{p} \cong \frac{E_{p}}{Z_{\text {line }}+Z}
$$

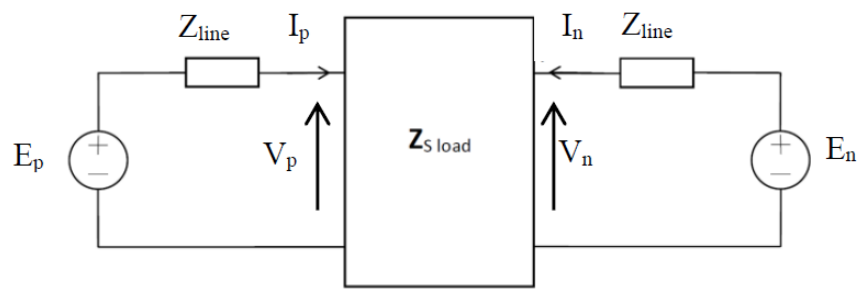

Figure 1. Coupling of the positive sequence and negative sequence circuits due to an asymmetrical three-phase load.

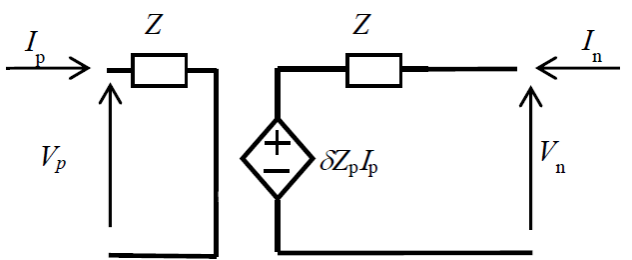

Figure 2. Approximate circuit representation of the impact of the positive sequence circuit on the negative sequence circuit, based on the weak coupling assumption.

The rigorous solution for $I_{p}$ in Fig. 1, however, is given by:

$$
I_{p}=\frac{E_{p}-\frac{\delta Z_{n}}{Z+Z_{\text {line }}} E_{n}}{Z+Z_{\text {line }}-\frac{\delta Z_{p} \delta Z_{n}}{Z+Z_{\text {line }}}}
$$

By comparing (14) and (15) we obtain the conditions for weak coupling assumption:

$$
\begin{gathered}
\left|\frac{\delta Z_{n}}{Z+Z_{\text {line }}} E_{n}\right| \ll\left|E_{p}\right| \\
\left|\frac{\delta Z_{p} \delta Z_{n}}{\left(Z+Z_{\text {line }}\right)^{2}}\right| \ll 1
\end{gathered}
$$

The constraint (16) is usually met since in most of practical cases the source is such that $\left|E_{n}\right| \ll\left|E_{p}\right|$. The constraint (17) should be checked, but it is reasonably expected that it is true in most of the cases where impedance deviations are small. However, when the three-phase load shows a large asymmetry (e.g., the case of a large single-phase load) the constraint (17) could be critical.

It is interesting to notice that even in the case of large impedance deviations, if such deviations are equal (i.e., $\delta Z_{a}=$ $\delta Z_{b}=\delta Z_{c}$ ) then $\delta Z_{p}=\delta Z_{n}=0$, which means that (17) is met. Moreover, if such deviations show a three-phase symmetry (i.e., $\delta Z_{a}, \delta Z_{b}, \delta Z_{c}$ with equal magnitude and $2 \pi / 3$ relative phase displacement) then $\delta Z_{p}=0$ or $\delta Z_{n}=0$.

In case of weak coupling, the positive sequence circuit can be solved by neglecting circuit coupling (i.e., by using (14)). Then, according to (11), the impact on the negative sequence circuit can be represented by a current-controlled voltage source $\delta Z_{p} I_{p}$ (see Fig. 2).

Finally, according to Figs. 1 and 2, the approximate expression for the Voltage Unbalance Factor (VUF) based on the weak coupling assumption can be easily derived [18]:

$$
V U F=\left|\frac{V_{n}}{V_{p}}\right| \cong\left|\frac{\delta z_{p}}{Z} \cdot \frac{z_{\text {line }}}{Z+Z_{\text {line }}}\right|
$$

\section{Statistical ANALYsis}

Asymmetrical loads often have unpredictable nature, i.e., they are characterized by parameters affected by uncertainty. Therefore, it makes sense to investigate how the load uncertainties propagate to the $V U F$ defined in (18).

A thorough statistical analysis foresees assigning a probabilistic distribution to the load deviations $\delta Z_{a}, \delta Z_{b}, \delta Z_{c}$, 
(i.e., by treating them as random variables) and deriving the corresponding statistical properties of $V U F$ in (18) where $\delta Z_{p}$ is a function of $\delta Z_{a}, \delta Z_{b}, \delta Z_{c}$ according to (12).

To this aim, it is useful to rewrite (18) by putting into evidence the random factor $\left|\delta Z_{p}\right|$ normalized by the nominal impedance $Z$ :

$$
V U F=K \cdot\left|\delta Z_{p} / Z\right|
$$

where:

$$
K=\left|\frac{Z_{\text {line }}}{Z+Z_{\text {line }}}\right|=\left|\frac{1}{1+Z / Z_{\text {line }}}\right|
$$

is a deterministic factor.

According to (12), $\left|\delta Z_{p}\right|$ is a function of the three random variables $\delta Z_{a}, \delta Z_{b}, \delta Z_{c}$. In this paper we consider the simpler case of only one asymmetrical load, i.e., only one random variable among $\delta Z_{a}, \delta Z_{b}, \delta Z_{c}$. This choice allows putting into evidence the effects of one single-phase load deviating from its nominal value $Z$. Thus, let us treat $\delta Z_{a}$ as a random variable, and $\delta Z_{b}=\delta Z_{c}=0$. We obtain:

$$
\left|\frac{\delta z_{p}}{Z}\right|=\frac{1}{3} \sqrt{\left(\frac{\delta R_{a}}{|Z|}\right)^{2}+\left(\frac{\delta X_{a}}{|Z|}\right)^{2}}
$$

Notice that a similar expression would be obtained by treating either $\delta Z_{b}$ or $\delta Z_{c}$ as random variable since $|\alpha|=$ $\left|\alpha^{2}\right|=1$.

For the sake of simplicity, we can rewrite (21) as:

$$
w=\frac{1}{3} \sqrt{x^{2}+y^{2}}
$$

with

$$
x=\frac{\delta R_{a}}{|Z|}, \quad y=\frac{\delta X_{a}}{|Z|}, \quad w=\left|\frac{\delta Z_{p}}{Z}\right|
$$

Let us assume $x$ and $y$ as random variables with Gaussian distribution:

$$
x \sim N\left(\mu_{x} ; \sigma^{2}\right), \quad y \sim N\left(\mu_{y} ; \sigma^{2}\right)
$$

With the assumption (24), the random variable $w$ in (22) has a Rice distribution whose probability density function (PDF) is given by [19]-[21]:

$$
f_{w}(w)=\frac{9 w}{\sigma^{2}} \exp \left(-\frac{9 w^{2}+a^{2}}{2 \sigma^{2}}\right) I_{0}\left(\frac{3 w a}{\sigma^{2}}\right)
$$

where:

$$
a=\sqrt{\mu_{x}^{2}+\mu_{y}^{2}}
$$

and $I_{0}$ is the zero-order modified Bessel function of the first kind.

The PDF of VUF in (19) can be obtained by resorting to the theorem on transformation of random variables. By denoting as $v$ the random variable VUF we obtain:

$$
f_{v}(v)=\frac{1}{K} f_{w}\left(\frac{v}{K}\right)
$$

Similarly, the cumulative distribution function (CDF) of the Rice random variable $w$ is given by:

$$
F_{w}(w)=1-Q_{1}\left(\frac{a}{\sigma}, \frac{3 w}{\sigma}\right)
$$

where $Q_{1}$ is the first-order Marcum Q-function.

Therefore, the CDF of $v$ (i.e., the CDF of the VUF) is given by:

$$
F_{v}(v)=F_{w}\left(\frac{v}{K}\right)
$$

The mean value of (19) can be obtained by multiplying by $K$ the mean value of the Rice random variable $w$ :

$$
\mu_{v}=\frac{K}{3} \sqrt{\frac{\pi}{2}} \sigma \cdot L_{1 / 2}\left(-\frac{a^{2}}{2 \sigma^{2}}\right)
$$

where $L_{1 / 2}$ is a Laguerre polynomial.

The variance of (19) can be obtained by multiplying by $K^{2}$ the variance of the Rice random variable $w$ :

$$
\sigma_{v}^{2}=\frac{K^{2}}{9}\left[2 \sigma^{2}+a^{2}-\frac{\pi}{2} \sigma^{2} L_{1 / 2}^{2}\left(-\frac{a^{2}}{2 \sigma^{2}}\right)\right]
$$

\section{NUMERICAL VALIDATION}

The statistical properties (27), (29)-(31) of the approximate expression (18) based on the weak coupling assumptions (16)(17) were validated by means of the numerical simulation of a simple radial network consisting in a $12.47 \mathrm{kV}-60 \mathrm{~Hz}$ threephase ideal source with only positive sequence component, a $3.22 \mathrm{~km}$ symmetrical line, and an asymmetrical star-connected load. The line was characterized by the following impedance matrix [17]:

$$
\tilde{\mathbf{Z}}=\left[\begin{array}{lll}
0.2494+j 0.8748 & 0.0592+j 0.4985 & 0.0592+j 0.4985 \\
0.0592+j 0.4985 & 0.2494+j 0.8748 & 0.0592+j 0.4985 \\
0.0592+j 0.4985 & 0.0592+j 0.4985 & 0.2494+j 0.8748
\end{array}\right]\left[\frac{\Omega}{k m}\right]
$$

whereas the nominal impedance of the load was $Z=10+$ j10 $\Omega$.

According to (23)-(24), the deviation $\delta Z_{a}$ of the load $Z_{a}$ with respect to the nominal value $Z$ was described by the normalized Gaussian random variables $x$ and $y$. For the sake of simplicity, it was assumed $\mu_{x}=\mu_{y}=\mu$ where $\mu$ ranges from 0 to 0.1 , and $\sigma$ was set to 0.01 . A repeated-run analysis was performed to obtain the numerical estimates of the PDF, CDF, mean value and standard deviation of the $V U F$ given by the exact solution of the coupled circuit in Fig. 1, i.e., 


$$
V U F=\left|\frac{V_{n}}{V_{p}}\right|=\left|\frac{-Z_{\text {line }} I_{n}}{E_{p}-Z_{\text {line }} I_{p}}\right|
$$

where $I_{p}$ is given by (15) and $I_{n}$ is evaluated accordingly. Such numerical estimates are compared with the corresponding approximate analytical results (27) and (29)(31).

In Fig. 3 the numerical and analytical PDFs (dotted and solid lines, respectively) are shown for six different values of $\mu$, i.e., $\mu=0,0.02,0.04,0.06,0.08,0.1$. By increasing $\mu$, i.e., the load asymmetry, larger values of $V U F$ are obtained. Numerical and approximate analytical PDFs (given by (27)) are clearly in good agreement.

Fig. 4 shows the behavior of the numerical and analytical CDFs for the same three values of $\mu$ considered in Fig. 3. Also in this case the analytical curves (given by (29)) are in good agreement with numerical results.

Figs. 5 and 6 correspond to Figs. 3 and 4, respectively, by assuming a larger value of the load standard deviation, i.e., $\sigma=0.02$ instead of 0.01 .

Figs. 7 and 8 correspond to Figs. 3 and 4 by assuming $\sigma=$ 0.04 . Clearly, by increasing the load standard deviation both the mean value and the standard deviation of $V U F$ are affected.

Fig. 9 shows the behavior of the $V U F$ mean value as a function of the mean value $\mu$ of the load asymmetry. Numerical results (dashed lines) are compared with approximate analytical results given by (30). Three different values for the load standard deviation were considered, i.e., $\sigma=0.01, \sigma=0.02, \sigma=0.04$. The curves are related to the PDFs shown in Figs. 3, 5, and 7.

Fig. 10 shows the behavior of the $V U F$ standard deviation as a function of the mean value $\mu$ of the load asymmetry as in Fig. 9. Numerical results (dashed lines) are compared with approximate analytical results given by the square root of (31). Three different values for the load standard deviation were considered, i.e., $\sigma=0.01, \sigma=0.02, \sigma=0.04$. The curves are related to the PDFs shown in Figs. 3, 5, and 7.

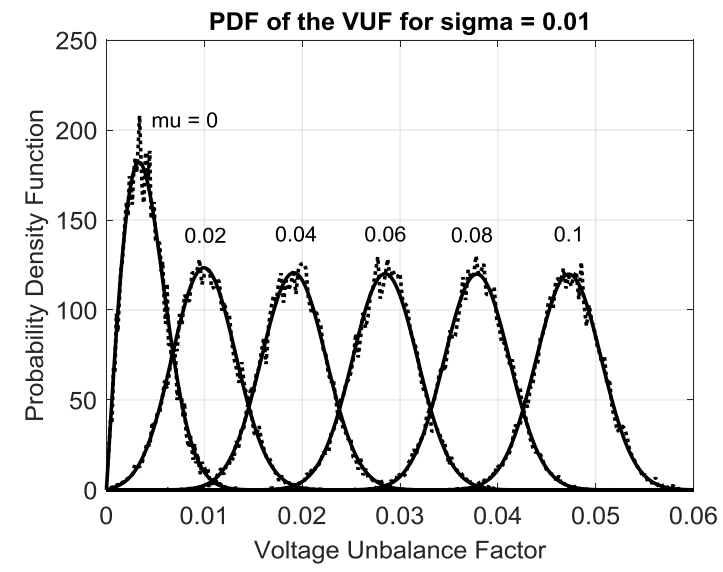

Figure 3. Probability density function of the voltage unbalance factor for different values of the load asymmetry mean value $\mu$, and for load asymmetry standard deviation $\sigma=0.01$. Analytical results (solid lines) are compared with numerical simulations (dotted lines).

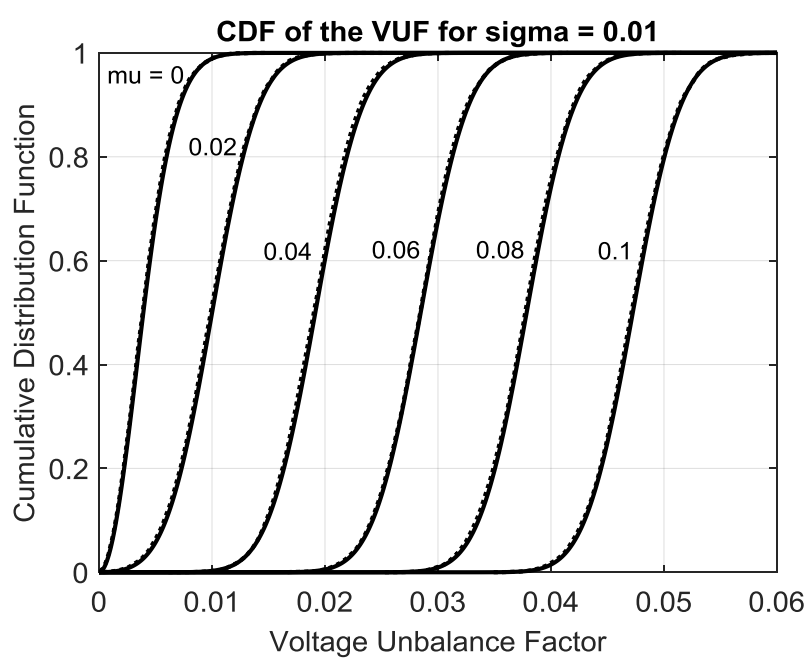

Figure 4. Cumulative distribution function of the voltage unbalance factor for different values of the load asymmetry mean value $\mu$, and for load asymmetry standard deviation $\sigma=0.01$. Analytical results (solid lines) are compared with numerical simulations (dotted lines).

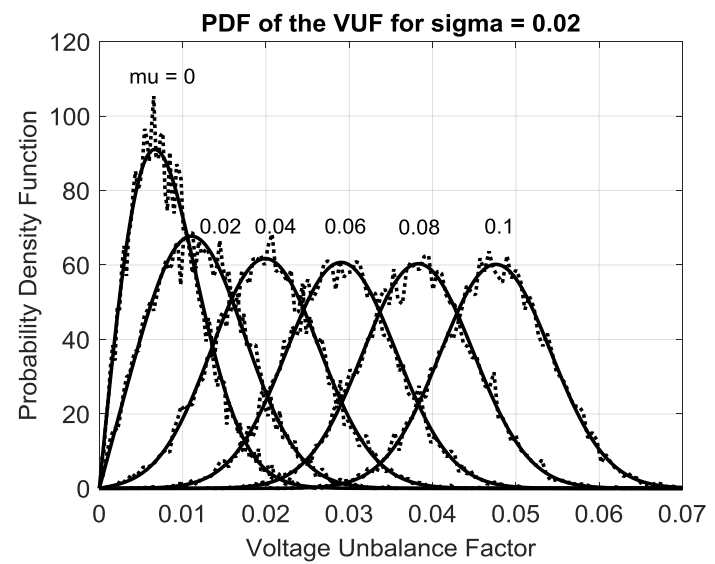

Figure 5. Probability density function of the voltage unbalance factor for different values of the load asymmetry mean value $\mu$, and for load asymmetry standard deviation $\sigma=0.02$. Analytical results (solid lines) are compared with numerical simulations (dotted lines).

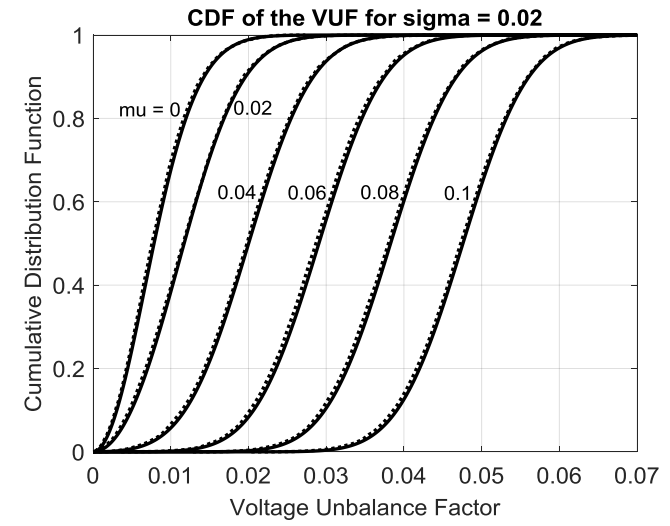

Figure 6. Cumulative distribution function of the voltage unbalance factor for different values of the load asymmetry mean value $\mu$, and for load asymmetry standard deviation $\sigma=0.02$. Analytical results (solid lines) are compared with numerical simulations (dotted lines). 


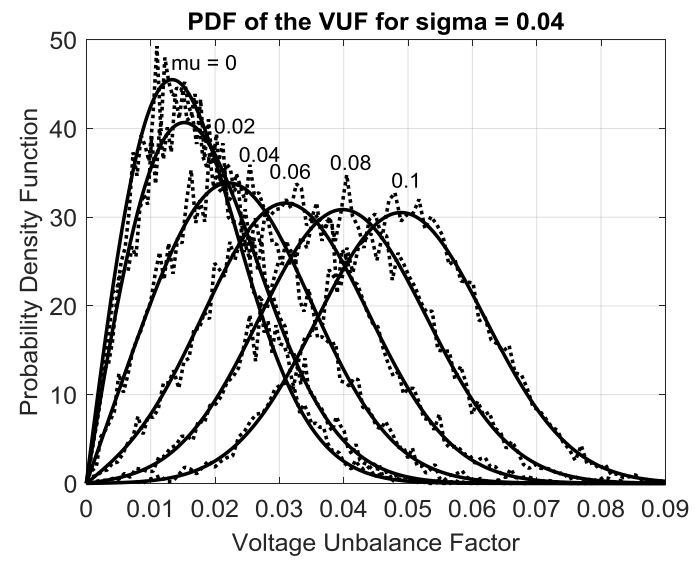

Figure 7. Probability density function of the voltage unbalance factor for different values of the load asymmetry mean value $\mu$, and for load asymmetry standard deviation $\sigma=0.04$. Analytical results (solid

lines) are compared with numerical simulations (dotted lines).

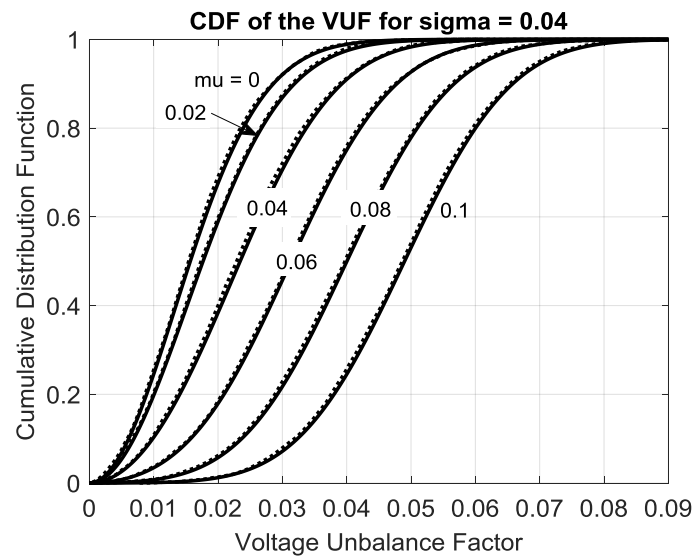

Figure 8. Cumulative distribution function of the voltage unbalance factor for different values of the load asymmetry mean value $\mu$, and for load asymmetry standard deviation $\sigma=0.04$. Analytical results (solid lines) are compared with numerical simulations (dotted lines).

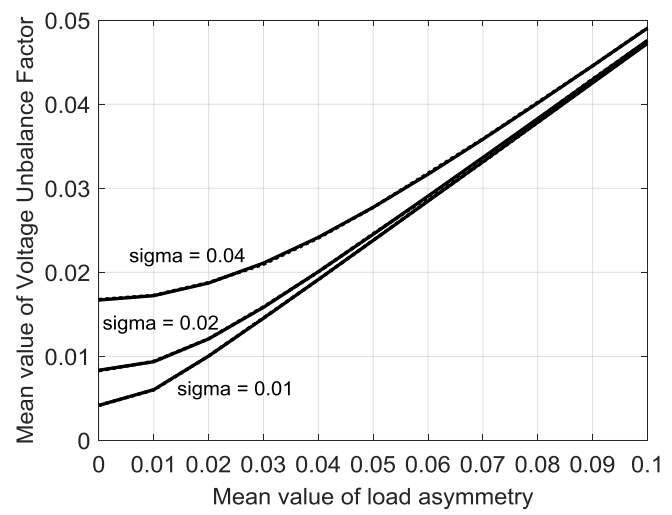

Figure 9. Mean value of the voltage unbalance factor as a function of the load asymmetry mean value $\mu$, for different values of the load asymmetry standard deviation $\sigma$. Analytical results (solid lines) are compared with numerical simulations (dotted lines).

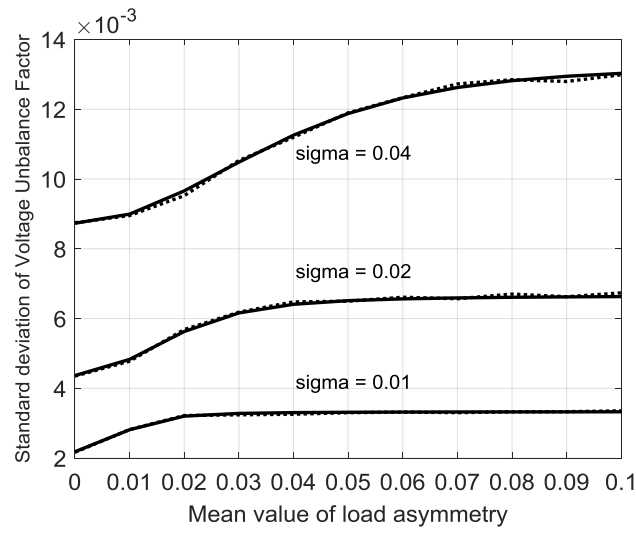

Figure 10. Standard deviation of the voltage unbalance factor as a function of the load asymmetry mean value $\mu$, for different values of the load asymmetry standard deviation $\sigma$. Analytical results (solid lines) are compared with numerical simulations (dotted lines).

\section{CONCLUSION}

An approximate model for the voltage unbalance emission based on the weak coupling assumption for the positive and negative sequence circuits in a three-phase power system with asymmetrical load has been derived. It was shown that under proper hypothesis a load asymmetry can be represented as a current-controlled voltage source in the negative sequence circuit.

The statistics of the voltage unbalance factor have been derived in closed form by assuming Gaussian distribution of the asymmetrical load parameters. In particular, the PDF, $\mathrm{CDF}$, mean value and variance of the VUF have been derived in closed form as functions of the uncertainty in one impedance in a three-phase load.

Future work will be devoted to extend the proposed approach to the more general case where all the load parameters are treated as random variables. Moreover, the analysis will be extended to the case where more than one three-phase load is asymmetrical. In that case, the statistical approach should allow a proper quantitative description in terms of superposition of voltage unbalance emissions.

\section{References}

[1] C. L. Fortescue, "Method of symmetrical coordinates applied to the solution of polyphase networks," Trans. AIEE, vol. 37, pp. 1027-1140, 1918.

[2] G. Chicco, P. Postolache, and C. Toader, "Analysis of three-phase systems with neutral under distorted and unbalanced conditions in the symmetrical componentbased framework," IEEE Trans. on Power Delivery, vol. 22, no.1, pp. 674-683, Jan. 2007.

[3] G. C. Paap, "Symmetrical components in the time domain and their application to power network calculations," IEEE Trans. on Power Systems, vol. 15, no. 2, pp. 522528, May 2000.

[4] D. Bellan and S. A. Pignari, "A circuit approach for the propagation analysis of voltage unbalance emission in power systems," in Proc. 2016 IEEE PES Asia Pacific 
Power and Energy Engineering Conference (APPEEC 2016), pp. 1355-1359.

[5] D. Bellan, G. Superti Furga, and S. A. Pignari, "Circuit representation of load and line asymmetries in three-phase power systems," International Journal of Circuits, Systems and Signal Processing, vol. 9, pp. 75-80, 2015.

[6] D. Bellan and S. A. Pignari, "Circuit representation of voltage unbalance emission due to line asymmetry," in Proc. 2015 IEEE Innovative Smart Grid Technologies Asia (ISGT ASIA), pp. 1-5.

[7] R. Muzzammel and U. Tahir, "Analytical behaviour of line asymmetries in three phase power systems," in Proc. of IEEE International Symposium on Recent Advances in Electrical Engineering, 24-26 October, 2017, Islamabad, Pakistan.

[8] J. Jayachandran and R. Murali Sachithanandam, "Implementation of Neural Network Based I $\cos \varphi$ Controller for DSTATCOM in Three Phase Four Wire Distribution System Under Varying Source and Load Conditions for Power Quality Improvement," WSEAS Transactions on Systems and Control, vol. 11, pp. 199216, 2016.

[9] T. Ahmadi, "A Novel Multi Stage Transformer for Compensating Unbalanced Loads," WSEAS Transactions on Systems and Control, vol. 15, pp. 282-286, 2020.

[10]Z. Emin and D. S. Crisford, "Negative phase-sequence voltages on E\&W transmission system," IEEE Trans. on Power Delivery, vol. 21, no. 3, pp. 1607-1612, July 2006.

[11]P. Paranavithana, S. Perera, R. Koch, and Z. Emin, "Global voltage unbalance in MV networks due to line asymmetries," IEEE Trans. on Power Delivery, vol. 24, no. 4, pp. 2353-2360, Oct. 2009.

[12] T. E. Seiphetlho and A. P. J. Rens, "On the assessment of voltage unbalance," in Proc. $201014^{\text {th }}$ International Conference on Harmonics and Quality of Power, pp. 1-6.

[13] H. Renner, "Voltage unbalance emission assessment," in Proc. Elect. Power Quality Supply Reliability Conf., June 2010, pp. 43-48.

[14] U. Jayatunga, S. Perera, and P. Ciufo, "Voltage unbalance emission assessment in radial power systems," IEEE Trans. Power Delivery, vol. 27, no. 3, pp. 1653-1661, July 2012.

[15] U. Jayatunga, S. Perera, P. Ciufo, and A. P. Agalgaonkar, "Deterministic methodologies for the quantification of voltage unbalance propagation in radial and interconnected networks," IET Generation, Transmission \& Distribution, vol. 9, no. 11, pp. 1069-1076, 2015.

[16] Electromagnetic compatibility (EMC) - limits assessment of emission limits for the connection of unbalanced installations to $M V, H V$ and EHV power systems, Technical report IEC/TR 61000-3-13, Ed. I, International Electrotechnical Commission, 2008.

[17]M. Olofsson and U. Grape, "Voltage quality in the context of EMC," in Proc. 2009 International Symposium on Electromagnetic Compatibility, Kyoto, Japan, pp. 241244.

[18]D. Bellan, "Approximate Circuit Representation of Voltage Unbalance Emission Due to Load Asymmetry in Three-Phase Power Systems," in Proc. of the 4th IEEE Global Electromagnetic Compatibility Conference
(GEMCCON), Stellenbosch, South Africa, 7-9 November, 2018, pp. 1-4

[19]D. Bellan, "Probability density function of three-phase ellipse parameters for the characterization of noisy voltage sags," IEEE Access, vol. 8, pp. 185503-185513, 2020.

[20]D. Bellan, "Circuit modeling and statistical analysis of differential-to-common-mode noise conversion due to filter unbalancing in three-phase motor drive systems," Electronics, vol. 9, pp. 1-20, 2020.

[21]D. Bellan, "Characteristic Function of Fundamental and Harmonic Active Power in Digital Measurements Under Nonsinusoidal Conditions," International Review of Electrical Engineering, vol. 10, no. 4, pp. 520-527, 2015.

\section{Creative Commons Attribution License 4.0 (Attribution 4.0 International, CC BY 4.0)}

This article is published under the terms of the Creative Commons Attribution License 4.0

https://creativecommons.org/licenses/by/4.0/deed.en_US 